\title{
A model for the turbulent Hartmann layer
}

T. Alboussière, and R. J. Lingwood

Citation: Physics of Fluids 12, 1535 (2000); doi: 10.1063/1.870402

View online: https://doi.org/10.1063/1.870402

View Table of Contents: http://aip.scitation.org/toc/phf/12/6

Published by the American Institute of Physics

\section{Articles you may be interested in}

On the stability of the Hartmann layer

Physics of Fluids 11, 2058 (1999); 10.1063/1.870068

On the measurement of the Hartmann layer thickness in a high magnetic field

Physics of Fluids 16, 3243 (2004); 10.1063/1.1768871

Interaction of a small permanent magnet with a liquid metal duct flow Journal of Applied Physics 112, 124914 (2012); 10.1063/1.4770155

Asymptotic analysis and symmetry in MHD convection

Physics of Fluids 8, 2215 (1996); 10.1063/1.868994

Kolmogorov flow in three dimensions

Physics of Fluids 9, 164 (1997); 10.1063/1.869159

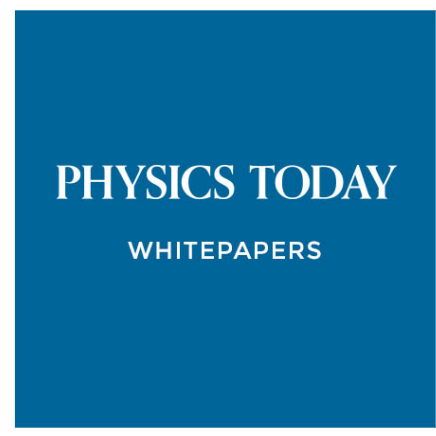




\title{
A model for the turbulent Hartmann layer
}

\author{
T. Alboussière ${ }^{\text {a) }}$ and R. J. Lingwood \\ Department of Engineering, University of Cambridge, Trumpington Street, Cambridge CB2 1PZ, \\ United Kingdom
}

(Received 25 March 1999; accepted 9 February 2000)

\begin{abstract}
Here we study the Hartmann layer, which forms at the boundary of any electrically-conducting fluid flow under a steady magnetic field at high Hartmann number provided the magnetic field is not parallel to the wall. The Hartmann layer has a well-known form when laminar. In this paper we develop a model for the turbulent Hartmann layer based on Prandtl's mixing-length model without adding arbitrary parameters, other than those already included in the log-law. We find an exact expression for the displacement thickness of the turbulent Hartmann layer [also given by Tennekes, Phys. Fluids 9, 1876 (1966)], which supports our assertion that a fully-developed turbulent Hartmann layer of finite extent exists. Leading from this expression, we show that the interaction parameter is small compared with unity and that therefore the Lorentz force is negligible compared with inertia. Hence, we suggest that the turbulence present in the Hartmann layer is of classical type and not affected by the imposed magnetic field, so justifying use of a Prandtl model. A major result is a simple implicit relationship between the Reynolds number and the friction coefficient for the turbulent Hartmann layer in the limit of large Reynolds number. By considering the distance over which the stress decays, we find a condition for the two opposite Hartmann layers in duct flows to be isolated (nonoverlapping). (C) 2000 American Institute of Physics. [S1070-6631(00)00906-5]
\end{abstract}

\section{INTRODUCTION}

The Hartmann layer is a fundamental element of magnetohydrodynamics (MHD). It develops along any boundary in an electrically-conducting fluid where the magnetic field is not tangential to the boundary, and it is where most of the shear stress is concentrated. The Hartmann layer provides a path for electrical currents that close within the core of the flow; thus controlling the whole flow. The state (i.e., whether laminar or turbulent) of the Hartmann layer is important because it affects the transfer of heat or mass through the layer and affects the global electric circulation. The experimental results reported in the literature (e.g., Hartmann and Lazarus, ${ }^{1}$ Murgatroyd, ${ }^{2}$ Lykoudis, ${ }^{3}$ and Branover $^{4}$ ) are generally dedicated to determining the friction coefficient for duct flows in the presence of a transverse magnetic field, from which the state of the flow may be determined. In our recent paper ${ }^{5}$ we consider the stability of the laminar Hartmann layer to both infinitesimal and finite-amplitude disturbances. Here, we are concerned with the profiles of both velocity and mean stress for an isolated Hartmann layer when it is turbulent. We consider the simplest configuration, which consists of uniform free-stream velocity above a plane boundary with an imposed nonparallel uniform magnetic field.

An example of an application in which the state of the Hartmann layers may be of importance is metallurgy and, in particular, steel casting, where steady magnetic fields are used. In this application, the nature of the flow is not well known but the velocities are large, of the order of a meter per

\footnotetext{
a) Author to whom correspondence should be addressed. Tel: (44) 1223332 645; fax: (44) 1223332 662; electronic mail: ta209@eng.cam.ac.uk
}

second. In such cases, the state of the Hartmann layers may be of primary importance in determining the global damping effect of the magnetic field. In fusion-reactor projects, a socalled liquid-metal blanket surrounds the plasma and is subjected to an intense magnetic field of several Tesla. The natural convection, which develops due to the large heat flux received, produces large velocities and possibly leads to turbulent Hartmann layers. The state of Hartmann layers are also of relevance to the liquid metal flows within sliding electric power contacts; see Ref. 6 where the Reynolds number (see Sec. II for the definition of $R$ ) ranges from about 0.18 to $1.4 \times 10^{3}$. Finally, the case of MHD- generated twodimensional turbulence is linked to the state of the Hartmann layer. It is generally assumed that the layer is laminar and therefore simply provides a "frictional" linear damping force (proportional to the velocity in the core) on the twodimensional core turbulence. If, however, the Hartmann layer is turbulent, this linear term should be replaced by another model. It is therefore important that the turbulent form of the Hartmann layer is well understood so that a turbulent model of the damping can be developed.

The structure of the paper is as follows: In Sec. II we define the configuration. Section III gives an exact description of the displacement thickness, which confirms that found by Tennekes, ${ }^{7}$ and Sec. IV is devoted to application of Prandtl's model, which results in a relationship between the friction coefficient and the Reynolds number. In Sec. V we develop an asymptotic expression for the friction coefficient in terms of a simple implicit formula, and in Sec. VI we compare our model with experimental results. These results are followed by a discussion and conclusions in Sec. VII and VIII, respectively. 


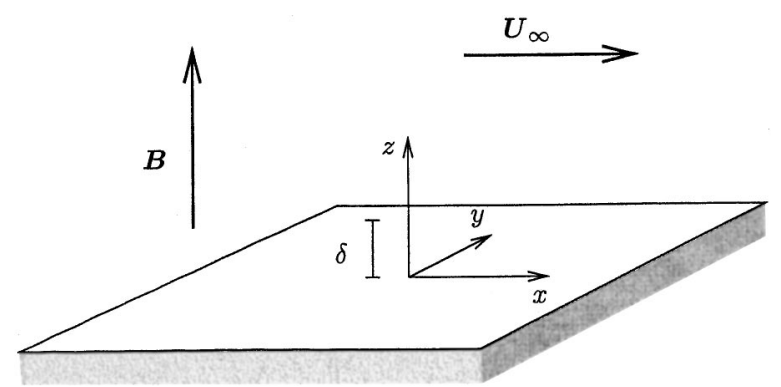

FIG. 1. The studied configuration.

\section{CONFIGURATION}

A similar configuration to that considered in Ref. 5 is examined here under the same assumption of the small magnetic Reynolds number. A plane boundary is perpendicular to a uniform magnetic field $B \mathbf{e}_{z}$ (where $\mathbf{e}_{z}$ is the unit vector along the $z$-axis). Sufficiently far above the boundary the flow is uniform and parallel to the boundary and is denoted by $U_{\infty} \mathbf{e}_{x}$ (see Fig. 1). This free-stream flow is also characterized by a uniform electric-current density that, combined with the magnetic field, opposes any free-stream pressure gradient. Therefore, as discussed in Ref. 5 and as will be proved in this section, for the Hartmann layer the pressure gradient and free-stream electric-current density can be taken as zero without loss of generality. The length-scale $\delta$ $=1 / B \sqrt{\nu \rho / \sigma}$ can be formed from the kinematic viscosity $\nu$, density $\rho$, electric conductivity $\sigma$, and magnetic-field intensity $B$. The same dimensional scales as those in Ref. 5 are chosen for velocity, time, pressure, magnetic field, electriccurrent density, and electric field potential, namely, $U_{\infty}$, $\delta / U_{\infty}, \rho U_{\infty}^{2}, B, \sigma U_{\infty} B$, and $U_{\infty} B \delta$, respectively. The momentum equation, mass continuity, Ohm's Law and electriccharge conservation take the following dimensionless forms:

$$
\begin{aligned}
& \frac{\partial \mathbf{u}}{\partial t}+(\mathbf{u} \cdot \boldsymbol{\nabla}) \mathbf{u}=-\boldsymbol{\nabla} p+\frac{1}{R} \mathbf{j} \wedge \mathbf{e}_{z}+\frac{1}{R} \nabla^{2} \mathbf{u}, \quad \boldsymbol{\nabla} \cdot \mathbf{u}=0, \\
& \mathbf{j}=-\boldsymbol{\nabla} \phi+\mathbf{u} \wedge \mathbf{e}_{z}, \quad \boldsymbol{\nabla} \cdot \mathbf{j}=0,
\end{aligned}
$$

where $\mathbf{u}, p, \mathbf{j}$, and $\phi$ are the dimensionless velocity, pressure, electric-current density, and electric potential fields, and where the Reynolds number $R=U_{\infty} \delta / \nu$ is the single dimensionless parameter. This number is the ratio of the Reynolds number $\mathrm{Re}=U_{\infty} H / \nu$ to Hartmann number $\mathrm{Ha}=\sqrt{\sigma /(\rho \nu)} B H$; whatever length scale $H$ is chosen $R$ $=\mathrm{Re} / \mathrm{Ha}$.

The laminar solution to this problem has exponential form decaying over the length-scale $\delta$, which is also the displacement thickness. In dimensionless form, this solution is

$$
\begin{aligned}
& \mathbf{u}=[u(z), 0,0]=\left[1-e^{-z}, 0,0\right], \\
& \mathbf{j}=[0, j(z), 0]=\left[0, e^{-z}, 0\right] .
\end{aligned}
$$

In the turbulent regime, provided that a finite-thickness fullydeveloped solution exists, the mean velocity is assumed to be in the direction of $\mathbf{e}_{x}$ and to depend on $z$ only. Reynolds decomposition is introduced to separate each quantity into a mean part (denoted with an overbar) and a fluctuating part (denoted with prime): $\mathbf{u}=\overline{\mathbf{u}}+\mathbf{u}^{\prime}, \mathbf{j}=\overline{\mathbf{j}}+\mathbf{j}^{\prime}, p=\bar{p}+p^{\prime}$, and $\phi=\bar{\phi}+\phi^{\prime}$. The mean part of the $x$-component of the dimensionless Navier-Stokes equation and of the $y$-component of Ohm's Law can be written as

$$
\begin{aligned}
& 0=-\frac{\partial \bar{p}}{\partial x}+\frac{\partial \bar{\tau}}{\partial z}+\frac{\bar{J}_{y}}{R}, \\
& \bar{J}_{y}=-\frac{\partial \bar{\phi}}{\partial y}-\bar{u}_{x},
\end{aligned}
$$

where $\bar{\tau}=-\overline{u_{x}^{\prime} u_{z}^{\prime}}+\left(\partial \bar{u}_{x} / \partial z\right) / R$ is the mean stress composed of Reynolds and viscous stresses. Substituting the expression for the electric-current density (5) into the momentum Eq. (4), we obtain

$$
0=-\frac{\partial \bar{p}}{\partial x}+\frac{\partial \bar{\tau}}{\partial z}-\frac{1}{R} \frac{\partial \bar{\phi}}{\partial y}-\frac{\bar{u}_{x}}{R} .
$$

If far from the boundary a uniform velocity is reached, (6) becomes

$$
0=-\frac{\partial \bar{p}_{\infty}}{\partial x}-\frac{1}{R} \frac{\partial \bar{\phi}_{\infty}}{\partial y}-\frac{1}{R},
$$

where $\bar{p}_{\infty}$ and $\bar{\phi}_{\infty}$ are the dimensionless pressure and electric potential in the free-stream. Under the assumption of invariance along $x$ and $y$, the gradients of $\bar{p}$ and $\bar{\phi}$ parallel to the boundary cannot depend on $z$ (therefore, for example, $\partial \bar{p} / \partial x=\partial \bar{p}_{\infty} / \partial x$ ), so subtracting (7) from (6) leads to the following simple expression for the momentum equation in the Hartmann layer:

$$
\frac{\partial \bar{\tau}}{\partial z}=\frac{1}{R}\left(\bar{u}_{x}-1\right) .
$$

This equation simply expresses the balance between stress and the Lorentz force. The magnetic field has been considered to be strictly perpendicular to the boundary. However, it can be shown that the above equation is unchanged for an inclined magnetic field (provided it is not parallel to the boundary) so long as the normal component of the magnetic field is used as the dimensional scale instead of the global magnitude of the magnetic field. Note that (8) was also derived by Tennekes, ${ }^{7}$ who used it to find an expression for the displacement thickness in a similar way to that described in the following section.

\section{AN EXACT RESULT FOR THE DISPLACEMENT THICKNESS}

Integrating (8) along $z$ between 0 and $\infty$ leads to the following expression for the dimensionless mean stress at the wall $\bar{\tau}_{w}$ :

$$
\bar{\tau}_{w}=\frac{1}{R} \int_{0}^{\infty} 1-\bar{u}_{x} d z .
$$

Alternatively, $\bar{\tau}_{w}$ can be written as the square of the dimensionless classical friction velocity $u_{*}$ or, equivalently, as half the friction coefficient $c_{f}$. The integral on the right-hand side of (9) is the dimensionless displacement thickness $\delta^{*}$ and so (9) can be written as 


$$
\delta^{*}=R \bar{\tau}_{w}=R u_{*}^{2}=\frac{R c_{f}}{2} .
$$

This result supports our assumption that a fully-developed turbulent Hartmann layer of finite thickness exists. This significant result appears, to our knowledge, in only one other paper $^{7}$ and seems to have been ignored since then. It is an exact expression and is independent of the state of the layer (i.e., whether laminar or turbulent); in the laminar regime, because $\delta^{*}=1$ (the displacement thickness $\delta$ is made dimensionless using itself), the expression leads to the well-known result; $c_{f}=2 / R=2 \mathrm{Ha} / \mathrm{Re}$.

More importantly, (10) can be used as follows to show that the turbulence present in the Hartmann layer must be of classical type and not affected by the magnetic field. We wish to define a Stuart number $N$ (or interaction parameter). It may seem natural to base this on $U_{\infty}$, in which case we find that $N=c_{f} / 2$ which is small at the scale of the turbulent Hartmann layer because the friction coefficient is expected to be, and indeed is found to be, much smaller than unity. Small Stuart number implies that the Lorentz force is negligible compared with inertia. However, to study the structure of the turbulence, it is more sensible to base the Stuart number on the turbulent velocity fluctuations, which are of order $U_{\infty} u_{*}$. This (turbulent) Stuart number is given by

$$
N=\frac{\sigma B^{2} \delta \delta^{*}}{\rho U_{\infty} u_{*}}=u_{*},
$$

which is still small compared with unity.

Based on this analysis, and contrary to previous investigators who tried to introduce an effect of the magnetic field on the structure of turbulence, we are justified in applying a simple conventional model for shear boundary layers, the Prandtl model.

\section{APPLICATION OF PRANDTL'S MODEL}

In our dimensionless variables, Prandtl's mixing-length model takes the form

$$
\frac{\partial \bar{u}_{x}}{\partial z}=2.5 \frac{\sqrt{\bar{\tau}}}{z} .
$$

The factor of 2.5 is traditionally used in the literature as it is thought to provide the best agreement between the log-law and experimental results. Note that this relationship (12) between stress and mean velocity must be replaced by a linear relationship in the viscous sublayer. The link between the classical $u^{+}$and $z^{+}$variables that are usually used in wallbounded turbulence and our dimensionless $\bar{u}_{x}$ and $z$ is

$$
\bar{u}_{x}=u_{*} u^{+}, \quad z=\frac{z^{+}}{u_{*} R} .
$$

Classically, the boundary of the viscous sublayer is taken to be $z^{+}=11.3$, where $u^{+}=11.3$ (see Ref. 8). (More precisely this distance $z^{+}=11.3$ lies within the intermediate zone between the viscous sublayer and the log-law region and should be considered as the virtual intersection of these two regions.) This will constitute an initial condition from which to integrate the coupled differential Eqs. (8) and (12) (and i)

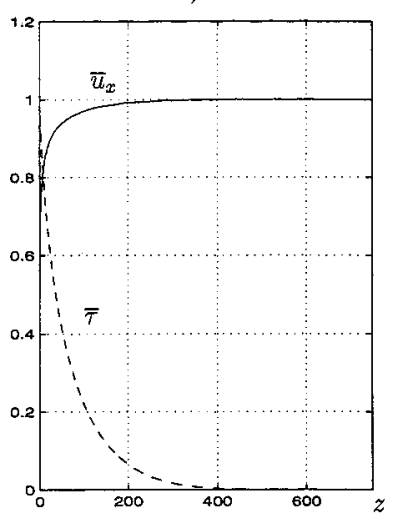

ii)

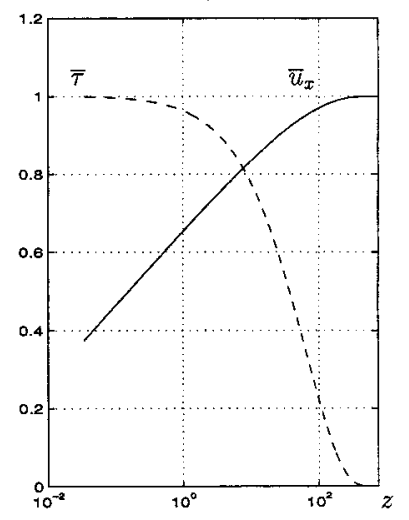

FIG. 2. The mean velocity and mean stress profiles in the turbulent Hartmann layer: (i) in linear coordinates, (ii) in semilogarithmic coordinates.

also using the fact that $\bar{\tau}=\bar{\tau}_{w}$ ), under the usual assumption that the viscous sublayer is so thin that it does not significantly contribute to the global momentum in the layer, and consequently does not significantly affect the stress. In our dimensionless variables, the initial conditions for the integration of the Prandtl model (12) together with the momentum equation (8) are

$$
\text { at } z=\frac{11.3}{u_{*} R}, \quad \bar{u}_{x}=11.3 u_{*} \text { and } \bar{\tau}=u_{*}^{2} .
$$

These equations have been integrated numerically using MATLAB. The parameter $R$ is known, whereas $u_{*}$ (or, equivalently, the friction at the wall) is unknown a priori and has to be determined. The condition used to determine $u_{*}$ is that in the limit of large $z$ the mean dimensionless velocity converges towards unity and that the mean stress converges towards zero (which are equivalent conditions). Numerically, the latter condition of vanishing stress at infinity proves more useful. For large $u_{*}$, the stress $\bar{\tau}$ never goes to zero; for small $u_{*}, \bar{\tau}$ goes to negative values, which are not physical. At the correct value for $u_{*}, \bar{\tau}$ decays monotonically towards zero at infinity. After 30 iterations of a bisection method, an accurate value of $u_{*}$ is found. In Fig. 2, the profiles of the converged solutions for $\bar{u}_{x}$ and $\bar{\tau}$ are given, in linear and semilogarithmic coordinates, for the particular value $R$ $=10^{4}$. It can be seen from the semilogarithmic plot that the initial point is $z \approx 3.41 \times 10^{-2}$ (corresponding to the end of the viscous sublayer), which corresponds to $11.3 /\left(R_{*} u_{*}\right)$ for the converged value of $u_{*} \approx 3.31 \times 10^{-2}$. It is interesting to compare the velocity curve with the classical log-law obtained under the condition of a uniform stress, $u^{+}$ $=2.5 \ln z^{+}+5.238$. (The numerical constant 5.238 results from the choice that the viscous sublayer should end at $z^{+}$ $=11.3$, so that the mean velocity profile is continuous from this layer to the log-law region, $11.3=2.5 \ln 11.3+5.238$.) These profiles are shown in Fig. 3.

This integration process has been performed for a range of values of $R$, between $R=300$ and $R=10^{6}$. It is then possible to plot the friction coefficient $c_{f}$ as a function of $R$. For small values of $R$ (typically less than 250), the Hartmann layer is thought to be entirely laminar (see Ref. 5, and the 


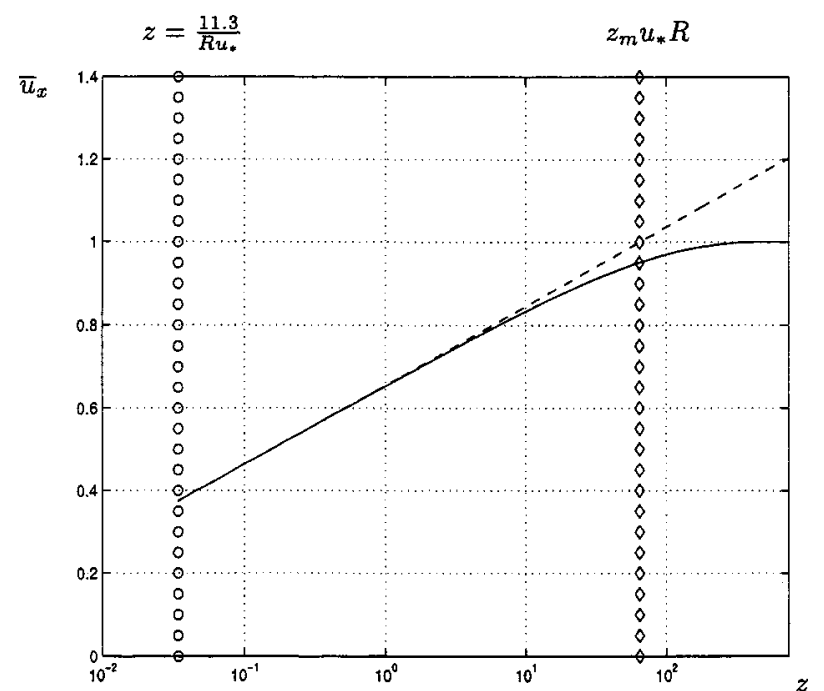

FIG. 3. The mean velocity profile at $R=10^{4}$ for the turbulent Hartmann layer $\bar{u}_{x}(-)$ in semilogarithmic coordinates compared with the log-law (---). The definition of $z_{m}$ is given by (26).

references therein, for details of the stability of the laminar Hartmann layer) and the friction coefficient is simply given by the viscous stress induced by the exponential mean velocity profile, i.e., by $c_{f}=2 / R$. The friction coefficient $\left(c_{f}\right.$ $\equiv 2 u_{*}^{2}$ ) is plotted in Fig. 4 in logarithmic coordinates for a range of Reynolds numbers including both the laminar and turbulent regimes. Although our model is probably less reliable at low values of $R$ because the viscous sublayer becomes non-negligible in thickness, it is nonetheless interesting to find the intersection of our curve with the laminar friction coefficient. It occurs at $R \approx 279$, a value very close to the "laminarization" value observed experimentally, see Ref. 5.

\section{ASYMPTOTIC-FRICTION EXPRESSION}

Although the integration presented in the previous section are not demanding in terms of computational resources,

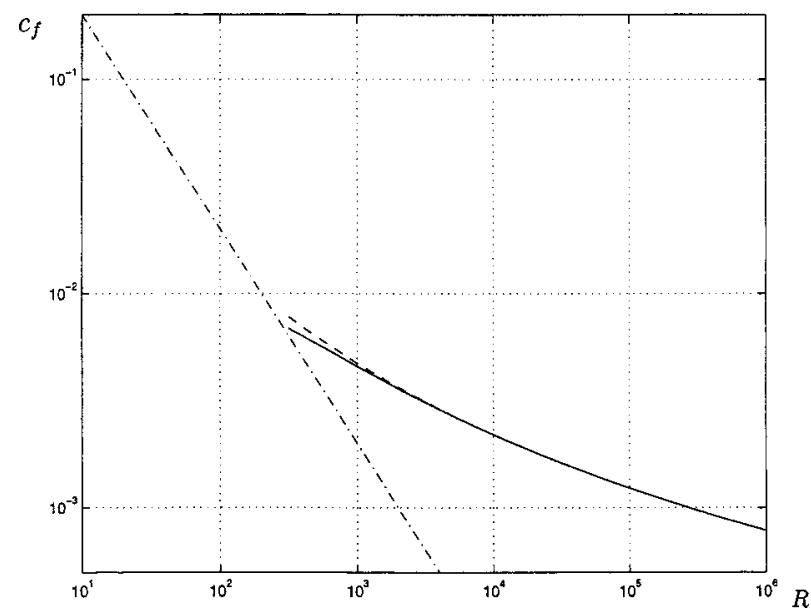

FIG. 4. The friction coefficient for the Hartmann layer $c_{f}$ in logarithmic coordinates in the laminar and turbulent regimes: ---, laminar model $\left(c_{f}\right.$ $=2 / R) ;$ - , application of Prandtl's model; ---, asymptotic model (28). it is convenient and enlightening to derive a simple formula for the friction coefficient. In this section, we obtain such a formula, which converges asymptotically towards the results given by the full Prandtl's model for large $R$.

During the numerical integrations, we observed that the function $\bar{\tau} / u_{*}^{2}$ converged towards a single asymptotic function when the parameter $R$ was increased, provided the $z$-coordinate was stretched in an appropriate way. This can be proved as follows. Differentiating (8) with respect to $z$ and substituting for $\partial \bar{u}_{x} / \partial z$ using (12), we obtain the following equation in $\bar{\tau}$ only:

$$
\frac{\partial^{2} \bar{\tau}}{\partial z^{2}}=\frac{2.5}{R} \frac{\sqrt{\bar{\tau}}}{z} .
$$

Introducing the new variables $f=\sqrt{\bar{\tau} / u_{*}^{2}}$ and $Z=z /\left(u_{*} R\right)$, this equation can be written as a differential equation in $f$ as

$$
\frac{\partial^{2} f^{2}}{\partial Z^{2}}=2.5 \frac{f}{Z}
$$

for which the initial condition is derived from (14) and the final condition is that $f$ vanishes when $Z$ goes to infinity. These conditions are given by

$$
\text { at } Z=\frac{11.3}{u_{*}^{2} R^{2}}, \quad f=1,
$$

when $Z \rightarrow \infty, \quad f \rightarrow 0$.

The parameter $R$ appears only in the initial condition for $f$. In fact, when $u_{*}^{2} R^{2} \gg 1$, the initial condition can be assumed to hold at $Z=0$. This is possible because $f$ can be extrapolated to $Z=0$. Indeed, integrating (16) twice in the neighborhood of $Z=0$ where $f \simeq 1$, we obtain

$$
f^{2}(Z) \simeq 2.5 Z \ln Z-2.5 Z+K Z+L,
$$

where $K$ and $L$ are constants. So when $R$ is large, and therefore the viscous sublayer is thin, we can consider that the initial condition applies at $Z=0$, and since the only dependence on $R$ then disappears, the function $f$ becomes universal (and independent of $R$ ). Thus the stress is expressed as

$$
\frac{\bar{\tau}}{u_{*}^{2}}=f^{2}\left(\frac{z}{u_{*} R}\right),
$$

where $f$ is the unique solution of the differential Eq. (16) with boundary conditions $f(0)=1$ and $f(Z \rightarrow \infty) \rightarrow 0$.

Now, (12) can be integrated. Note that the integration for velocity must start at the edge of the viscous sublayer (not at zero) because close to the wall the velocity in the turbulent region has approximately logarithmic form and physically cannot be extrapolated to zero. Thus, integration of (12), with initial velocity given by the velocity at the edge of the viscous sublayer, gives

$$
\bar{u}_{x}=11.3 u_{*}+2.5 \int_{11.3 / u_{*} R}^{z} \frac{u_{*} f\left(z^{\prime} /\left(u_{*} R\right)\right)}{z^{\prime}} d z^{\prime} .
$$

When $z$ goes towards infinity, the velocity approaches the free-stream velocity $\left(\bar{u}_{x} \rightarrow 1\right)$. In this limit the previous equation gives 


$$
\frac{1}{u_{*}}=11.3+2.5 \int_{11.3 / u_{*}^{2} R^{2}}^{\infty} \frac{f(Z)}{Z} d Z,
$$

where the integration variable has been changed to $Z$ $=z /\left(u_{*} R\right)$. It is interesting to split the integral into three terms such that

$$
\begin{aligned}
\frac{1}{u_{*}}= & 11.3+2.5 \int_{1}^{\infty} \frac{f(Z)}{Z} d Z+2.5 \int_{11.3 / u_{*}^{2} R^{2}}^{1} \frac{f(Z)-1}{Z} d Z \\
& +2.5 \int_{11.3 / u_{*}^{2} R^{2}}^{1} \frac{1}{Z} d Z .
\end{aligned}
$$

The first integral is a constant (independent of $R$ and $c_{f}$ ). The second integral converges towards a constant value when $u_{*}^{2} R^{2}$ approaches infinity; to prove this, we just need to show that $(f(Z)-1) / Z$ can be integrated at zero. From (19) near $Z=0, f$ can be written as $f(Z) \simeq \sqrt{1+2.5 Z \ln Z \text {, }}$ which yields

$$
\frac{f(Z)-1}{Z} \simeq \frac{2.5}{2} \ln Z,
$$

in the neighborhood of $Z=0$, which can be integrated. The third integral can be readily calculated. In the limit of large $u_{*}^{2} R^{2}$, (23) takes the form

$$
\frac{1}{u_{*}}=A+2.5 \ln \left(\frac{u_{*}^{2} R^{2}}{11.3}\right)
$$

where $A$ is a constant. Including $A$ partially in the logarithm, this can equivalently be written as

$$
\frac{1}{u_{*}}=2.5 \ln \left(z_{m} u_{*}^{2} R^{2}\right)+5.238,
$$

where $z_{m}$ is a constant, which is shown in Fig. 3. The value of $z$ at the intersection of the log-law curve (for the same friction velocity as the computed profile) with the line $\bar{u}_{x}$ $=1$, is seen numerically to converge towards $0.1938 u_{*} R$, when $R$ increases towards infinity. Hence, the constant $z_{m}$ is close to 0.1938 and (26) can be written as

$$
\frac{1}{u_{*}}=2.5 \ln \left(0.1938 u_{*}^{2} R^{2}\right)+5.238,
$$

which constitutes a simple implicit equation relating $u_{*}$ to $R$. If $u_{*}$ is replaced by its counterpart $c_{f}$, this equation becomes

$$
\sqrt{\frac{2}{c_{f}}}=2.5 \ln \left(0.0969 c_{f} R^{2}\right)+5.238 .
$$

This simple relationship is plotted in Fig. 4. At $R=300$, the discrepancy between this relationship and the result of our application of Prandtl's model (see Sec. IV) is about $14 \%$. It drops quickly with increasing $R$ [due to the increase of $u_{*}^{2} R^{2}$, which constitutes the condition of asymptotic validity of (28)]: when $R=500$ the relative discrepancy is $8 \%$, at $R$ $=1000$ it is $3.4 \%$, at $R=2000$ it is $1.4 \%$, and at $R=5000$ it is only $0.4 \%$. At large values of $R$, the asymptotic curve merges perfectly with the result of the full Prandtl's model.

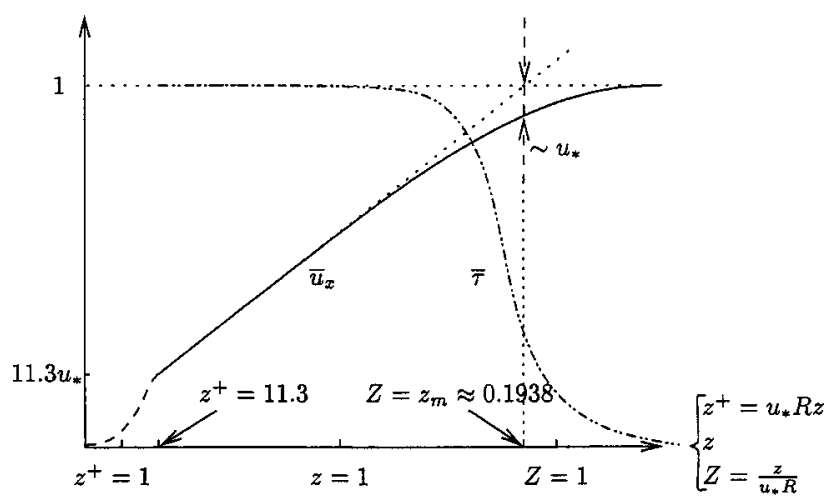

FIG. 5. Sketch of the structure of the turbulent Hartmann layer in semilogarithmic coordinates.

Integration of Prandtl's model, with the condition of vanishing stress at infinity, has resulted in the structure sketched in Fig. 5. The position of the intersection of the tangent $\log$-velocity profile with the free-stream velocity, i.e., at $Z=z_{m} \approx 0.1938$ seems to contradict the necessity that $\delta^{*}=u_{*}^{2} R\left(\ll u_{*} R\right)$. This can be resolved by proving that 1 $-\bar{u}_{x} \sim u_{*}$ at $Z=z_{m} \approx 0.1938$. From (21), because $\bar{u}_{x}$ converges to 1 when $z$ goes to infinity,

$$
1-\bar{u}_{x}=2.5 \int_{z_{m} u_{*} R}^{\infty} \frac{u_{*} f\left(z /\left(u_{*} R\right)\right)}{z} d z,
$$

which leads to

$$
1-\bar{u}_{x}=2.5 u_{*} \int_{z_{m}}^{\infty} \frac{f(Z)}{Z} d Z .
$$

This integral is a constant, so the velocity defect $\left(1-\bar{u}_{x}\right)$ is proportional to $u_{*}$ at the intersection position. A practical consequence is that when $R$ increases, the velocity profile is given by its asymptotes more and more closely.

If the interaction parameter is calculated based on $u_{*} R$ (the distance over which the stress decays), rather than based on the displacement thickness, then $N \sim 1$ : this results from our "non-MHD turbulence" analysis. This shows that the limit of our assumption that the interaction parameter is small is reached at the edge of the layer. This should not significantly affect our conclusions.

To summarize, the asymptotic treatment not only provides a simple relationship in good agreement with the detailed model, but it also tells us that the typical distance over which the stress decays is $u_{*} R$ (in the $z$-scale or, equivalently, unity in the $Z$-scale) and not $u_{*}^{2} R$, which is the displacement thickness as expressed in (10). The stress-decay scale will become important when assessing the domain of validity of our model when comparing with experiments in the following section.

\section{COMPARISON WITH EXPERIMENTS}

From the start of experimental MHD using liquid metals, the effect of applying a magnetic field to duct flows has been characterized by the measurement of pressure losses. Many experiments have been carried out essentially in the 60 e.g., Refs. $1,2,4,9,10,11$, on the pressure drop in circular and 


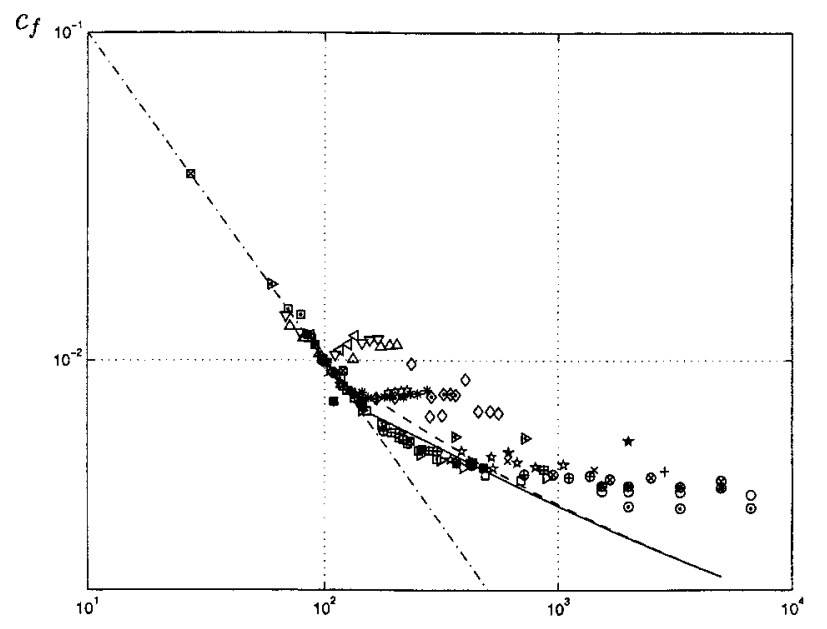

$R$

FIG. 6. The friction coefficient for the Hartmann layer $c_{f}$ vs $R$ in logarithmic coordinates in both the laminar (-.-) and turbulent ( - , application of Prandtl's model; ---, asymptotic model) regimes. See Fig. 8 for the key to the symbols representing the experimental data.

rectangular cross-sectional duct flows under transverse magnetic fields. In addition to the aspect ratio (for rectangular ducts), two dimensionless numbers characterize a given flow: the Reynolds number $\operatorname{Re}=U a / \nu$, where $a$ denotes the half-width in the direction of the magnetic field and where $U$ is an average velocity, and the Hartmann number $\mathrm{Ha}$ $=\sqrt{\sigma /(\rho \nu)} B a$. If the velocity profile is relatively flat due to the presence of the magnetic field or due to its turbulent nature, the average velocity $U$ is close to the velocity in the middle of the cavity. We are particularly interested in the case of rectangular ducts with the magnetic field aligned with the short dimension because then the friction characteristics are likely to come only from the two Hartmann layers on the two opposite walls, with the parallel layers playing an insignificant role. In this case, the friction coefficient has the same value whether it is defined as we have defined $c_{f}$ or as $-a \partial p / \partial x /\left(\rho U^{2} / 2\right)$, which is the definition used in the experimental literature. The results obtained by Hartmann and Lazarus, ${ }^{1}$ Murgatroyd, ${ }^{2}$ Brouillette and Lykoudis ${ }^{9}$ and Branover et al. ${ }^{4,10,11}$ are shown in Fig. 6 in logarithmic coordinates [i.e., $\ln c_{f}$ vs $\ln (\mathrm{Re} / \mathrm{Ha}) \equiv \ln R$ ] and in Fig. 7 as $c_{f}$ in terms of $2 \mathrm{Ha} / \mathrm{Re} \equiv 2 / R$, where $R$ is the Reynolds number based on displacement thickness that we have used thus far in our study of the Hartmann layer. Note that the laminar friction law derived by Hartmann and Lazarus $^{1}\left(c_{f}=2 / R\right)$ is included in these figures.

Our analysis can be applied only to the case when the two Hartmann layers on opposite walls of the duct do not overlap. As seen in Sec. V, the dimensionless decay distance for the stress in the turbulent Hartmann layer scales as $u_{*} R$. From our numerical solutions we notice that the distance $u_{*} R / 2$ corresponds to significant decay. We adopt this distance and thus we take the condition for the Hartmann layers not to overlap to be

$$
\frac{\mathrm{Re}}{\mathrm{Ha}^{2}} u_{*}<1,
$$

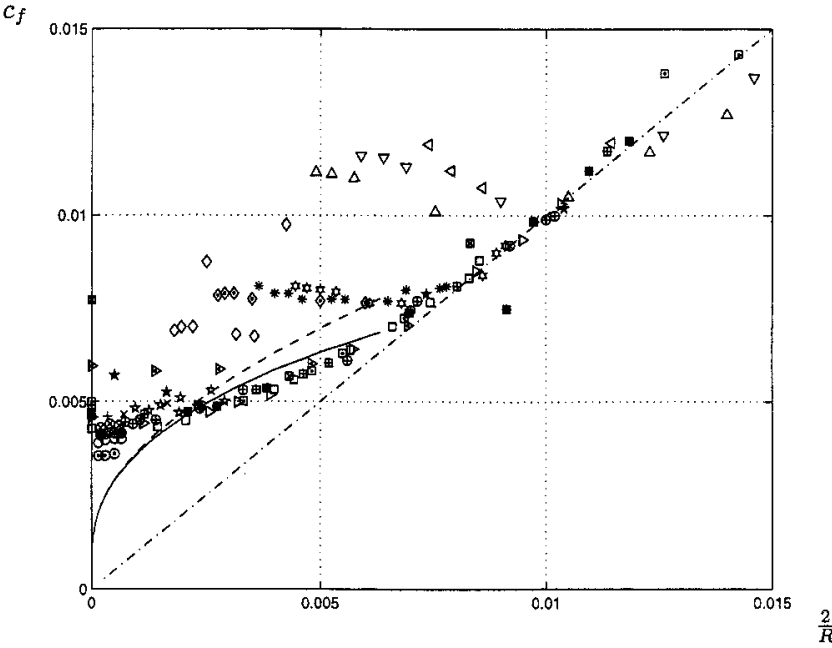

FIG. 7. The friction coefficient of a Hartmann layer $c_{f}$ vs $2 / R$ in laminar (-.-) and turbulent (-, application of Prandtl's model; ---, asymptotic model) regimes (same points as in Fig. 6).

where $u_{*}$ is a function of $R=\mathrm{Re} / \mathrm{Ha}$. Using our asymptotic friction law (27), this condition can be plotted in the $\mathrm{Re}-\mathrm{Ha}$ plane (Fig. 8) and is replaced by the curve $\mathrm{Re} / \mathrm{Ha}=250$ at low Reynolds numbers. All the experimental points below this boundary cannot be compared with our analysis because the Hartmann layers then overlap. In fact, our turbulent model is restricted to the region between the two curves; i.e., where the Hartmann layers do not overlap and where the Hartmann layers are turbulent (below $\mathrm{Re} / \mathrm{Ha} \approx 250$ turbulent duct flows are observed experimentally to laminarize; see Ref. 5).

Figure 9 is obtained from Fig. 7 after removing the points that do not satisfy our condition (31) or for which the Hartmann number is too small $(\mathrm{Ha}<10)$. (When the Hartmann number is less than 10 , the laminar friction formula $c_{f}=2 / R$ is not valid because then the laminar Hartmann layers overlap.) All the points fall on a single curve $c_{f}(R)$,

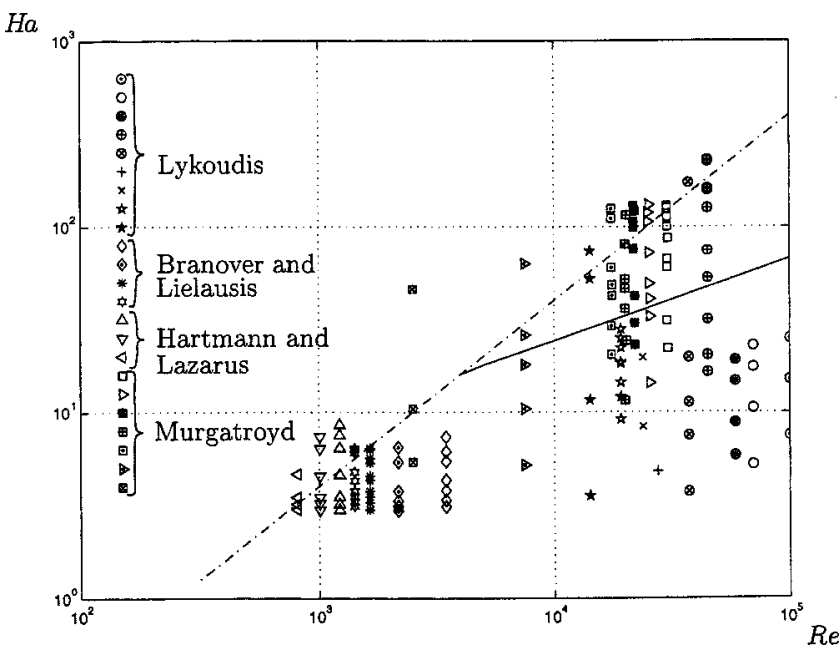

FIG. 8. Boundary in the Re-Ha plane above which the Hartmann layers do not overlap (-) and $\mathrm{Re} / \mathrm{Ha}=250$ (-.-) below which the Hartmann layers are turbulent. 


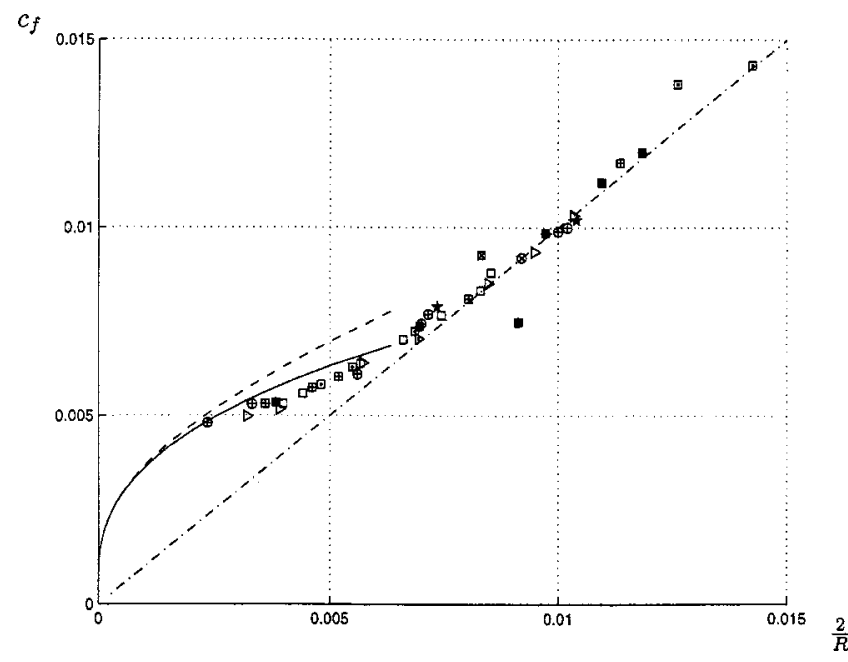

FIG. 9. The friction coefficient for the Hartmann layer $c_{f}$ vs $1 / R$ for the experimental data corresponding to isolated nonoverlapping Hartmann layers.

conform to the laminar friction at low values of $R$ and are in good agreement with our model at large values of $R$.

We conclude this section by assessing our model versus local experimental measurements. There have been very few experimental studies taking velocity profiles or velocityfluctuation measurements in MHD duct flows. However, we note that Branover et al. ${ }^{10}$ mention briefly that when the Re is increased (or Hartmann number decreased, i.e., with increasing $R$ ) the fluctuations (of electric potential) appear first close to the walls and then spread inwards affecting the whole cavity. This feature suggests that the Hartmann layers become turbulent before the core flow and determine the global pressure drop in the duct, which qualitatively supports our model. This suggestion is contrary to the accepted view, which is that the global flow controls the transition process. Some mean velocity profiles can be found in Brouillette and Lykoudis, ${ }^{9}$ where a standard Pitot-tube method was used. The reported accuracy is of order $5 \%$. We have selected the two profiles among theirs that satisfy our condition of nonoverlapping [see (31)] turbulent Hartmann layers (i.e., $\mathrm{Re} / \mathrm{Ha}>250)$ and plotted them in Figs. 10 and 11 together with our model prediction. The $z$-range in these figures corresponds to the half-width of the duct but, rather than nondimensionalizing with the duct dimension, we have used our dimensionless $z$-coordinate. The experimental measurements shown in Figs. 10 and 11 start at some distance from the wall, which means that there is limited comparison with our model in the near-wall region, however the agreement over the remaining ranges of $z$ is good.

\section{COMPARISON WITH OTHER THEORIES}

Our model is based on a finite-thickness assumption for the fully-developed turbulent Hartmann layer, which in fact is proved by relation (10) (initially published by Tennekes ${ }^{7}$ ). A primary consequence of this is that there is only one dimensionless parameter governing the flow, $R=\mathrm{Re} / \mathrm{Ha}$. According to Tennekes, Shercliff ${ }^{12}$ first expressed this condition in the form that the friction coefficient should be a function

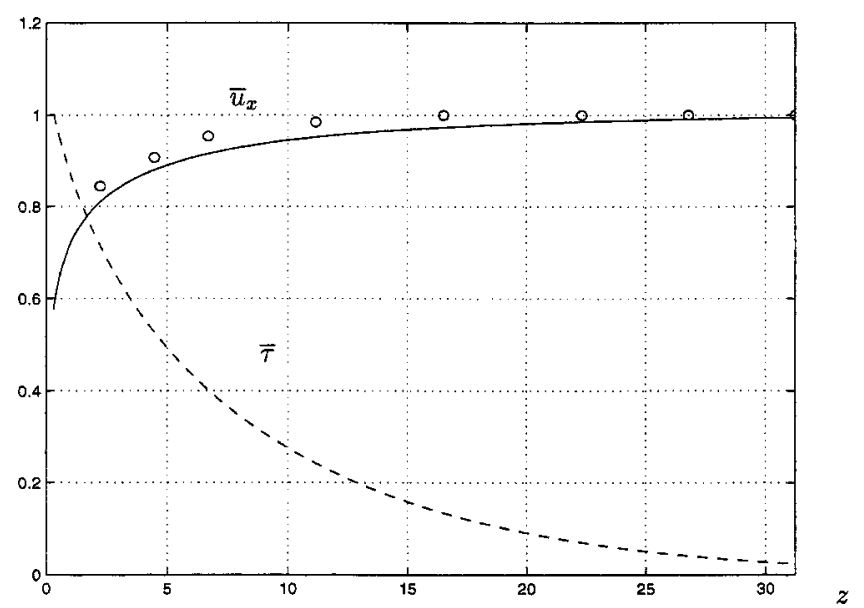

FIG. 10. Comparison of our theory with an experimental velocity profile from Ref. 9 corresponding to nonoverlapping turbulent Hartmann layers $(\mathrm{Re}=43000, \mathrm{Ha}=62.5)$.

of the single parameter $R=\mathrm{Re} / \mathrm{Ha}$, and not a function of the two independent parameters $\mathrm{Re}$ and Ha. This criterion will be used to assess the different models proposed. A second consequence of (10) is that we have defined a turbulent interaction parameter (11), based on this displacement thickness, that is small. We are thus justified in our use of a classical Prandtl model for wall-bounded turbulence, with no additional assumptions.

A number of theories have been proposed in the literature for the effect of turbulence in Hartmann layers, although often these are presented as theories for turbulence in duct flows rather than the Hartmann layers themselves. The major existing theories are listed below along with a short description and assessment.

(1) Hartmann and Lazarus (Ref. 1). At the end of this pioneering paper, the authors propose a simplified model for the effect of a transverse magnetic field on turbulence in duct flows. They consider the drag and attribute two opposite effects to the presence of the magnetic field; a reduction in drag due to the damping of turbulent fluc-

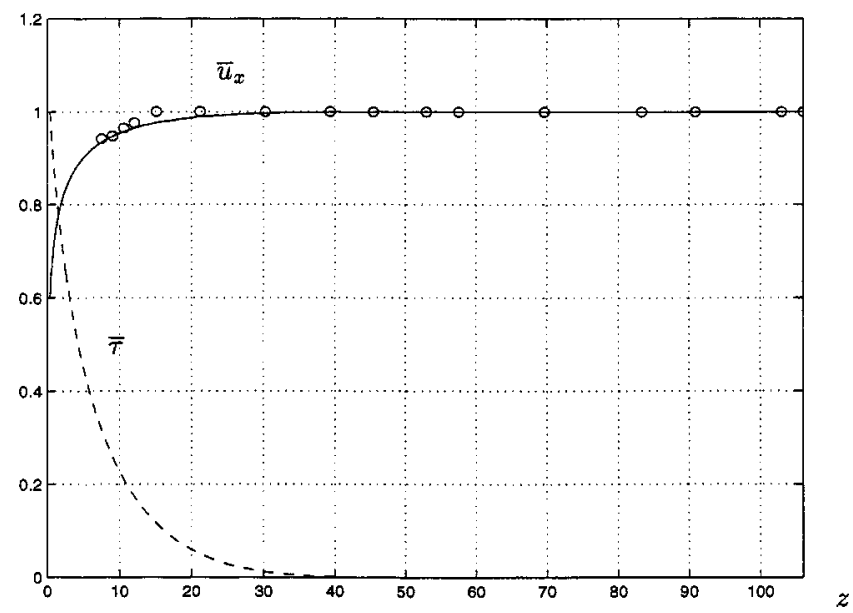

FIG. 11. Comparison of our theory with another experimental velocity profile from Ref. 9 corresponding to nonoverlapping turbulent Hartmann layers $(\mathrm{Re}=115000, \mathrm{Ha}=212)$. 
tuations and an increase in the drag due to the direct Lorentz braking force. This model is rather qualitative and considers the global flow; the parameter $R=\mathrm{Re} / \mathrm{Ha}$ is not identified as important.

(2) Maciulaitis and Loeffler (Ref. 13). An integral momentum equation for a Hartmann layer growing from a leading edge is considered. The model contains arbitrary parameters and relationship (10) is not satisfied.

(3) Branover (Ref. 4). A Prandtl model is considered, with an added assumption to account for effects of the magnetic field on the structure of the turbulence (a so-called "semiempirical theory"). The exact relationship (10) is not taken into account.

(4) Lykoudis (Ref. 3, 8). A similar theory to that developed by Branover, but with a different added assumption to account for the influence of the magnetic field on the Reynolds stress. Lykoudis admits that this phenomenological additional term cannot be justified rigorously (see footnote 20 of Ref. 8).

(5) Nihoul (Ref. 14). A completely independent approach based on Malkus' ideas on turbulence. An extremum principle on the shear-gradient distribution is invoked. Included in this model is the effect of an averaged Lorentz force and adjustable parameters to ensure continuity with results from the laminar regime. The results lead to an estimate for the turbulent Hartmann-layer thickness [not satisfying (10)] and to a relationship between $c_{f}$, Re and Ha; again $R=\mathrm{Re} / \mathrm{Ha}$ is not identified as important.

(6) Tennekes (Ref. 7). In a short paper, Tennekes derives equations equivalent to (8) and (10). In the rest of the paper, approximations are made. The dimensionless friction velocity $u_{*}$ is taken arbitrarily to be $1 / 20$. A analogy with no rigorous justification is made with the case of the boundary-layer flow with uniform suction at the boundary (and no magnetic field), and a relationship between $c_{f}$ and $R=\mathrm{Re} / \mathrm{Ha}$ is proposed in which they are inversely proportional at high $R$. This relationship does not seem to compare well with experimental results (Ref. 8).

In addition to these theories, some numerical computations have been performed and compared with experimental results; see, for example, Refs. 6, 15, 16. The numerics have shown that the turbulence is essentially concentrated in the Hartmann layers and that these regions grow in size when the Reynolds number is increased for a constant Hartmann number. This feature is also found in experiments.

\section{CONCLUDING REMARKS}

We are of the opinion that our model is not just one more model for the turbulent Hartmann layer to be added to the list given in the previous section. It has the advantage over the existing models that it requires no unjustifiable assumptions; only Prandtl's model with the usual coefficients leading to the log-law is considered. In that sense it is the simplest possible model for the turbulent Hartmann layer. Our model shows that the electrical conductance of the wall is irrelevant, as is the global electric circulation in the flow.
The state of the turbulent Hartmann layer is entirely dependent on the control parameter $R=\mathrm{Re} / \mathrm{Ha}$ only. It should also be noted that the most important property of the laminar Hartmann layer, namely, that the electric current flowing inside the layer is proportional to the velocity in the neighbouring core, is lost in the turbulent state.

Possible extensions of the work presented here, include application of a similar methodology to other fullydeveloped boundary layers of uniform thickness, e.g., the Ekman layer and the wall-bounded layer with uniform suction at the wall. The Ekman layer, although it is threedimensional, seems to correspond closely to the Hartmann layer, because the dominant Coriolis force is linear (like the electromagnetic Lorentz force in the present study). In the case of suction, the laminar mean-flow solution is identical to that for the laminar Hartmann layer, and the results of linear stability analysis are close to that of the Hartmann layer. Nevertheless, differences must exist in the turbulent states, if only because for suction the global momentum equation leads to $v_{0} U_{\infty}=-u_{*}^{2} U_{\infty}^{2}$ (where $v_{0}$ is the dimensional suction velocity). This relates $u_{*}$ to $U_{\infty}$, rather than to $\delta^{*}$ as in (10) for the Hartmann layer; $\operatorname{see}^{17}$.

In conclusion, we should say that MHD turbulence has different characteristics. According to our model, MHD turbulence in the turbulent Hartmann layer is of the classical type; the magnetic field simply playing the role of establishing the average electromagnetic Lorentz force. It is possible, likely even, that some turbulence with a tendency towards two-dimensionality exists in the core flow between the two opposite Hartmann layers (see the experimental evidence in Refs. 18 and 19). This quasi-two-dimensional turbulence, characterized by large interaction parameter, contributes very little to the stress, and exists because the mean electromagnetic Lorentz force is inefficient at damping it (note that there is no electric current in purely two-dimensional turbulence).

\section{ACKNOWLEDGMENT}

R.J.L. acknowledges the support provided by a Dorothy Hodgkin Royal Society Fellowship.

\footnotetext{
${ }^{1}$ J. Hartmann and F. Lazarus, "Experimental investigations on the flow of mercury in a homogeneous magnetic field,' K. Dan. Vidensk. Selsk. Mat. Fys. Medd. 15, 1 (1937).

${ }^{2}$ W. Murgatroyd, "Experiments on magnetohydrodynamic channel flow," Philos. Mag. 44, 1348 (1953).

${ }^{3}$ P. S. Lykoudis, "Transition from laminar to turbulent flow in magnetofluid mechanic channels," Rev. Mod. Phys. 32, 797 (1960).

${ }^{4}$ G. G. Branover, 'Resistance of magnetohydrodynamic channels,', Magnetohydrodynamics 3, 1 (1967).

${ }^{5}$ R. J. Lingwood and T. Alboussière, "On the stability of the Hartmann layer,' Phys. Fluids 11, 2058 (1999).

${ }^{6}$ G. Talmage, J. S. Walker, S. H. Brown, N. A. Sondergaard, H. Branover, and S. Sukoriansky, "Liquid-metal flows in sliding electrical contacts with arbitrary magnetic-field orientations,', Phys. Fluids A 3, 1657 (1991).

${ }^{7}$ H. Tennekes, "'Turbulent magnetohydrodynamic channel flow,' Phys. Fluids 9, 1876 (1966).

${ }^{8}$ P. S. Lykoudis and E. C. Brouillette, "Magneto-fluid-mechanics channel flow. II. Theory,', Phys. Fluids 10, 1002 (1967).

${ }^{9}$ E. C. Brouillette and P. S. Lykoudis, "Magneto-fluid-mechanics channel flow. I. Experiment,' Phys. Fluids 10, 995 (1967).

${ }^{10}$ G. G. Branover, Y. M. Gelfgat, and A. B. Tsinober, "Turbulent magne-
} 
tohydrodynamic flows in prismatic and cylindrical ducts,' Magnetohydrodynamics 2, 1 (1966).

${ }^{11}$ G. G. Branover, A. S. Vasilev, Y. M. Gelfgat, and E. V. Shcherbinin, "Turbulent flow in a plane perpendicular to a magnetic field," Magnetohydrodynamics 2, 46 (1966).

${ }^{12}$ J. A. Shercliff, The Theory of Electromagnetic Flow Measurement (Cambridge University Press, New York, 1962).

${ }^{13}$ A. Maciulaitis and A. L. Loeffler Jr., " A theoretical investigation of MHD entrance flows,' AIAA J. 2, 2100 (1964).

${ }^{14}$ J. C. J. Nihoul, "The Malkus theory applied to magnetohydrodynamic turbulent channel flow,' J. Fluid Mech. 25, 1 (1966).

${ }^{15}$ Y. Shimomura, "Large eddy simulation of magnetohydrodynamic turbu- lent channel flows under a uniform magnetic field,' Phys. Fluids A 3, 3098 (1991).

${ }^{16}$ S. Cuevas, B. F. Picologlou, J. S. Walker, G. Talmage, and T. Q. Hua, "Heat transfer in laminar and turbulent liquid-metal MHD flows in square ducts with thin conducting or insulating walls,' Int. J. Eng. Sci. 35, 505 (1997).

${ }^{17}$ H. Tennekes, "Similarity laws for turbulent boundary layers with suction or injection,' J. Fluid Mech. 21, 689 (1965).

${ }^{18} \mathrm{Yu}$. B. Kolesnikov and A. B. Tsinober, "Two-dimensional turbulence flow behind a circular cylinder,', Magnetohydrodynamics 8, 300 (1972).

${ }^{19} \mathrm{~J}$. Sommeria, "Experimental study of the two-dimensional inverse energy cascade in a square box,', J. Fluid Mech. 170, 139 (1986) 